Percutaneous transhepatic cholangiographic drainage (PTCD) catheters are the preferred method of decompressing obstructed bile ducts if endoscopic transpapillary stent placement is not possible. However, like transpapillary stents, transhepatic drainage catheters occlude frequently and have to be exchanged at regular intervals. Exchanging PTCDs poses a technical challenge to the gastroenterologist or radiologist.

We report here the case of a 70-year-old woman who had undergone a Whipple operation for pancreatic cancer 1 year previously. She subsequently developed malignant obstructive jaundice and, at another institution, had a PTCD placed from the external skin through the bile duct, with a choledochoenterostomy into the jejunum (Yamakawa-type prosthesis). She presented at our hospital with recurrent obstructive jaundice. The prosthesis was found to be occluded, and an attempt was made to exchange it by passing a straight guide wire through the prosthesis percutaneously. However, several attempts to pass the guide wire were unsuccessful. During the procedure, the patient had an upper endoscopy performed to locate the distal orifice of the prosthesis, in order to attempt retrograde passage of the guide wire. It was possible to locate the stent orifice $30 \mathrm{~cm}$ distal to the Billroth II anastomosis, but the guide wire could not be passed. The stent was then grasped with a rat-tooth forceps, and the Yamakawa prosthesis was withdrawn by gently pulling it from outside and advancing the forceps through the gastroscope under fluoroscopic guidance (Figure 1). Once the forceps appeared at the skin level, the new prosthesis was grasped and the forceps was pulled back into the gastroscope. Correct positioning of the prosthesis was verified by fluoroscopy and endoscopic visualization of free-flowing bile drainage. The patient tolerated the procedure well and was discharged from the hospital the next day.

\title{
An Alternative Method of Exchanging an Occluded Percutaneous Transhepatic Biliary Prosthesis (Yamakawa Type)
}
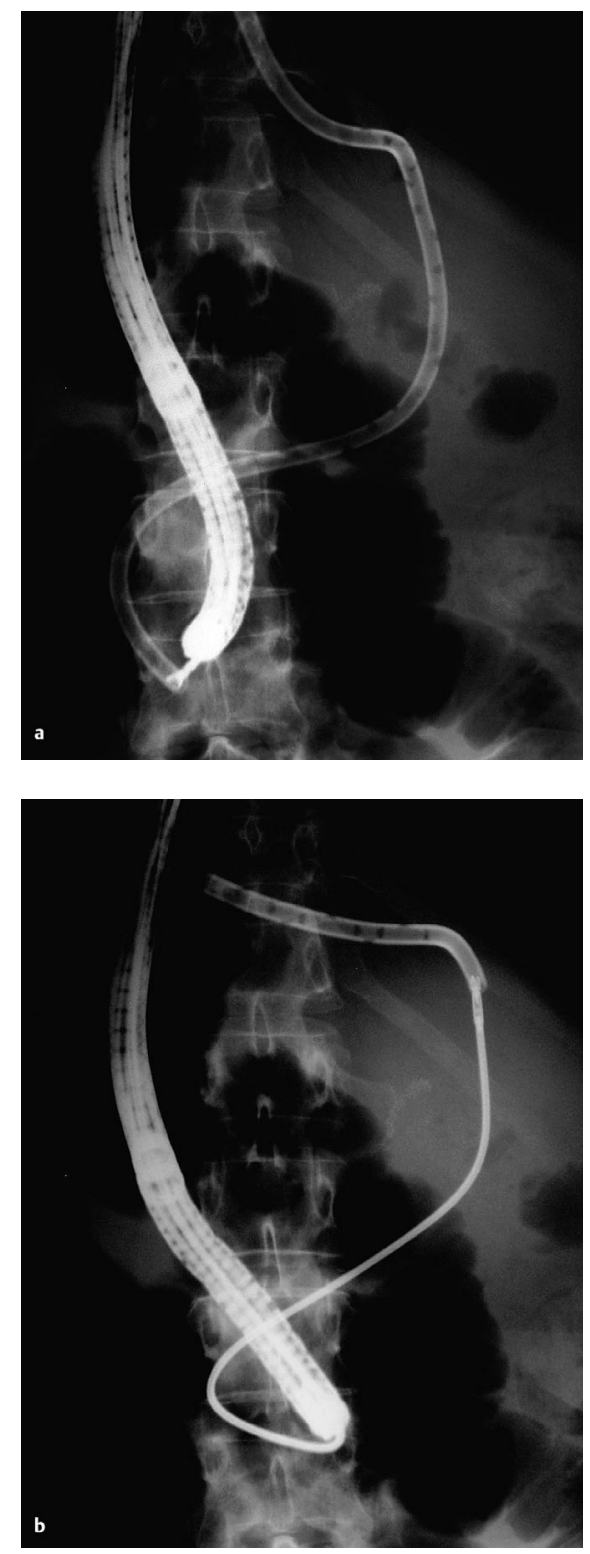

Figure 1 Fluoroscopy during the procedure. a The Yamakawa prosthesis is grasped with the rat-tooth forceps. b The prosthesis is withdrawn halfway through the liver, with the forceps following passively.

This new method of exchanging PTCDs represents an alternative to the regular method, which involves advancing a Jtipped guide wire through the prosthesis. The advantage of the method described here is that a (usually expensive) straight guide wire does not have to be bent into a J shape, rendering it useless for future en- doscopic applications. We would not currently recommend the method described here for routine care, but view it as a second-line approach. It should not be attempted if the prosthesis has not been in place for several weeks, since the tract may not have organized sufficiently for the forceps to be passed.

\section{S. Goebel ${ }^{1}$, M. Parusel ${ }^{2}$, B. Krakamp ${ }^{2}$ \\ ${ }^{1}$ Dept. of Internal Medicine II, Cologne-Merheim Hospital, Cologne City Hospitals, Cologne, Germany \\ ${ }^{2}$ Dept. of Internal Medicine I, Cologne-Merheim Hospital, Cologne City Hospitals, Cologne, Germany.}

\section{Corresponding Author}

\section{B. Krakamp, M.D.}

Klinik I für Innere Medizin

Kliniken der Stadt Köln

Krankenhaus Köln-Merheim

Ostmerheimer Str. 200

51109 Cologne

Germany

Fax: + 49-221-8907-3495

E-mail: krakampb@kliniken-koeln.de 